\title{
Análise comparativa entre os tratamentos para alopecia: uma revisão sistemática
}

\author{
Comparative analysis between treatments for alopecia: a systematic review \\ Análisis comparativo entre tratamientos para la alopecia: una revisión sistemática
}

Recebido: 03/11/2021 | Revisado: 11/11/2021 | Aceito: 12/11/2021 | Publicado: 21/11/2021

\author{
Brunna Michelly da Silva Sousa \\ ORCID: https://orcid.org/0000-0002-3187-3432 \\ Pontifícia Universidade Católica de Goiás, Brasil \\ E-mail: brunna.michelly@outlook.com \\ Thaís Jales Natal \\ ORCID: https://orcid.org/0000-0001-5018-9428 \\ Universidade em Porto Nacional, Brasil \\ E-mail: thaisjnatal@hotmail.com \\ Tamyres Borges Pereira \\ ORCID: https://orcid.org/0000-0002-9721-2506 \\ Pontifícia Universidade Católica de Goiás, Brasil \\ E-mail: tamyresborges94@gmail.com \\ Mariana Vieira Martins Sampaio Drummond \\ ORCID: https://orcid.org/0000-0002-6136-5535 \\ Universidade Federal de Goiás, Brasil \\ E-mail: maryy_drummond@hotmail.com \\ Roberpaulo Anacleto Neves \\ ORCID: https://orcid.org/0000-0003-1181-2373 \\ Pontifícia Universidade Católica de Goiás, Brasil \\ E-mail: roberpaulo_@hotmail.com
}

\begin{abstract}
Resumo
Introdução: A alopecia é a ausência temporária ou congênita de pelos ou cabelos, podendo ser classificada em cicatricial e não cicatricial. Esse estudo focou na análise comparativa dos tratamentos da alopecia areata e androgenética, as quais são alopecias não cicatriciais. A alopecia androgenética é a forma mais comum de perda de cabelo na espécie humana e a alopecia areata possui acometimento de cerca de $2 \%$ no âmbito mundial. Objetivo: $\mathrm{O}$ estudo visou conhecer, correlacionar e comparar as diferentes formas de tratamento para a alopecia areata e androgenética nos estudos mais recentes. Método: Trata-se de uma revisão sistemática de artigos científicos publicados na base de dados PUBMED entre 01/01/2018 e 31/12/2018. Foram usados os descritores "alopecia areata and treatment" e "alopecia androgenetic and treatment". Primeiro, os artigos foram analisados pelo título e resumo/abstract por dois revisores (BMSS e TJ), e em caso de desacordos, um terceiro revisor foi consultado (RAN). A seguir, houve uma leitura completa dos textos, e em caso de desacordos, um último revisor foi consultado (RAN). Resultados: A busca por "alopecia areata and treatment" resultou em 26 registros, dos quais 14 foram excluídos. Após a leitura dos artigos na íntegra, dentre os 12 artigos, apenas sete foram utilizados. A busca por "alopecia androgenetic and treatment" resultou em 84 registros, dos quais 61 artigos não atenderam aos critérios de inclusão. Após lidos os 24 artigos, apenas nove foram selecionados. Logo, após a fase de seleção, 16 artigos permaneceram elegíveis. Conclusão: Há uma vasta gama de tratamentos para a alopecia, mostrando que o tratamento deve ser individualizado para cada paciente. Deve-se lembrar sempre de associar a terapia farmacológica ao acompanhamento psicológico, uma vez que o cabelo é também é um instrumento de comunicação.
\end{abstract}

Palavras-chave: Alopecia; Tratamento; Revisão sistemática.

\begin{abstract}
Introduction: Alopecia is the temporary or congenital absence of hair or hair and can be classified as scarred and nonscarred. This study focused on the comparative analysis of the treatments of androgenetic alopecia areata, which are non-scarring alopecia. Androgenetic alopecia is the most common form of hair loss in humans and alopecia areata has around $2 \%$ worldwide. Objective: The study aimed to know, correlate and compare the different forms of treatment for andeatetic alopecia areata in the most recent studies. Method: This is a systematic review of scientific articles published in the PUBMED database between 01/01/2018 and 12/31/2018. The keywords "alopecia areata and treatment" and "alopecia androgenetic and treatment" were used. First, the articles were analyzed by title and abstract by two reviewers (BMSS and TJ), and in case of disagreement, a third reviewer was consulted (RAN). Afterwards, there was a complete reading of the texts, and in case of disagreement, a last reviewer was consulted (RAN). Results: The search for "alopecia areata and treatment" resulted in 26 records, of which 14 were excluded. After reading the full articles, of the 12 articles, only seven were used. The search for "alopecia androgenetic and treatment" resulted in out of 84 records, of which 61 articles did not meet the inclusion criteria.After reading the 24 articles, only nine were
\end{abstract}


selected.After the selection phase, 16 articles remained eligible. Conclusion: There is a wide range of treatments for alopecia, showing that the treatment should be individualized for each patient.Always remember to associate pharmacological therapy with psychological follow-up, since hair is also a communication tool.

Keywords: Alopecia; Therapy; Systematic review.

\section{Resumen}

Introducción: La alopecia es la ausencia temporal o congénita de cabello o cabello y se puede clasificar en cicatriz y sin cicatriz. Este estudio se centró en el análisis comparativo de tratamientos para la alopecia areata androgenética, que son alopecia no cicatricial. La alopecia androgenética es la forma más común de pérdida de cabello en los seres humanos y la alopecia areata tiene alrededor del $2 \%$ en todo el mundo. Objetivo: el estudio tuvo como objetivo conocer, correlacionar y comparar las diferentes formas de tratamiento de la alopecia areata anatética en los estudios más recientes. Método: Se trata de una revisión sistemática de artículos científicos publicados en la base de datos PUBMED entre el 01/01/2018 y el 31/12/2018. Se utilizaron las palabras clave "alopecia areata y tratamiento" y "alopecia androgenética y tratamiento". Primero, los artículos fueron analizados en términos de título y resumen por dos revisores (BMSS y TJ) y, en caso de desacuerdo, se consultó a un tercer revisor (RAN). Luego, los textos fueron leídos íntegramente y, en caso de desacuerdo, se consultó a un último revisor (RAN). Resultados: La búsqueda de "alopecia areata y tratamiento" resultó en 26 historias clínicas, de las cuales 14 fueron excluidas. Después de leer los artículos en su totalidad, de los 12 artículos, solo se utilizaron siete. La búsqueda de "alopecia androgenética y tratamiento" dio como resultado 84 historias clínicas, de las cuales 61 artículos no cumplieron con los criterios de inclusión. Después de leer los 24 artículos, solo se seleccionaron nueve. Después de la fase de selección, 16 artículos siguieron siendo elegibles. Conclusión: Los tratamientos para la alopecia son diversos, demostrando que el tratamiento debe ser individualizado para cada paciente, recuerde siempre asociar la terapia farmacológica con el seguimiento psicológico, ya que el cabello también es una herramienta de comunicación.

Palabras clave: Alopecia; Tratamiento; Revisión sistemática.

\section{Introdução}

O cabelo é um órgão constituinte do ser humano com função mecanossensorial, de termorregulação, proteção física, regeneração tecidual e comunicação psicossocial. Alguns distúrbios podem afetar essa estrutura, um deles é a alopecia (Oliveira \& Machado, 2017).

A alopecia é definida como a ausência temporária ou congênita de pelos ou cabelos de forma total ou parcial, acometendo principalmente o couro cabeludo, sendo mais comum em homens do que mulheres (Ejjiyar \& Driss El Amrani, 2018). A alopecia pode ser classificada em cicatricial e não cicatricial. A cicatricial é resultado de malformações, danos ou inflamações que levam à destruição de folículos pilosos por causas primárias ou secundárias. A alopecia não cicatricial, por sua vez, não é caracterizada por destruição dos folículos pilosos, e sim por redução desses, queda excessiva capilar ou interrupções de processos metabólicos que regulam o crescimento dos folículos, o que torna o cabelo mais curto, fino e despigmentado (Oliveira \& Machado, 2017).

Esse estudo foca no tratamento das alopecias não cicatriciais, mais especificamente da alopecia areata e androgenética. A alopecia androgenética é uma doença na qual a queda de cabelo ocorre devido à predisposição genética, que pode ocorrer em qualquer idade após a puberdade e em ambos os sexos. É uma alopecia dependente de andrógeno em indivíduos geneticamente predispostos, em que ocorre conversão dos fios em cabelos miniaturizados (Dhaher \& Yacoub, 2018).

A alopecia areata, por sua vez, é caracterizada por perda súbita de cabelo de etiologia obscura, mas quase sempre associada a outras doenças autoimunes (como tireoidites), o que sugere um processo autoimune antibulbo piloso. Associado a tal componente autoimune, há um componente genético que determina a predisposição de um indivíduo para sofrer fatores externos que podem atuar como gatilhos. No entanto, a exata etiologia e mecanismos patogênicos permanecem desconhecidos. Acomete igualmente ambos os sexos e geralmente aparece em adultos jovens e crianças (Dawber \& Van Neste, 1996; Gonul, et. al., 2018; Gomez-Zubiaur, et al., 2018; Thanomkitti, et al., 2018).

Quanto aos aspectos clínicos, na alopecia androgenética, há substituição gradual dos fios pigmentados e grossos por fios despigmentados e finos. A alopecia androgenética pode ter padrão de queda masculino ou feminino; no masculino, cursa 
com recessão bitemporal e calvície no vértice; no feminino, há afinamento difuso da parte superior e manutenção da linha do cabelo frontal (Dawber \& Van Neste, 1996).

A clínica da alopecia areata, por sua vez, caracteriza-se por perda súbita de cabelo com áreas de alopecia arredondadas ou ovais bem circunscritos, a placa é lisa e brilhante, apresentando em sua borda pelos peládicos (facilmente removíveis e afilados em direção à raiz), sendo que os cabelos brancos frequentemente são poupados. A evolução é favorável, ocorrendo crescimento capilar entre 2 a 6 meses. Apesar de essa clínica ser mais frequente no couro cabeludo, pode ocorrer também em outras áreas do corpo que apresentam pelos, como supercílios, cílios, barba e pelos pubianos (Dawber \& Van Neste, 1996; Carvalho \& D'Acri, 2014).

Segundo uma revisão sistemática publicada em 2018, a epidemiologia da alopecia areata corresponde a cerca de $2 \%$ no âmbito mundial, com ligeiro viés para o gênero feminino, isso devido à maior preocupação feminina em relação à perda de cabelo e à busca por tratamento. Verificou-se também que a incidência durante a vida aumentou a uma taxa quase linear e a mediana de idade do diagnóstico foi de 33 anos. Os homens tiveram maior probabilidade de diagnóstico na infância e as mulheres na adolescência (Darwin, 2018). Um outro estudo, também de 2018, mencionou que a alopecia androgenética é o tipo mais comum de alopecia que afeta mais de 50\% dos homens com idade acima de 40 anos e $75 \%$ das mulheres com idade acima de 65 anos (Faghihi, 2018). Publicado no mesmo ano, outro estudo afirmou que a alopecia androgenética é a forma mais comum de perda de cabelo em humanos, afetando $80 \%$ dos homens caucasianos e 50\% das mulheres caucasianas (MartinezJacobo, et al., 2018).

Com base no atual cenário epidemiológico, esse estudo é relevante por levantar as diversas linhas de tratamento da alopecia areata e androgenética, distúrbios que acometem os homens e as mulheres em alta proporção. Essa pesquisa visa conhecer, correlacionar e comparar as diferentes formas de tratamento para a alopecia areata e androgenética, as quais correspondem aos dois tipos de alopecia mais abordados nos estudos mais recentes.

\section{Metodologia}

Trata-se de uma revisão sistemática de artigos científicos publicados na base de dados PUBMED no período entre 01/01/2018 e 31/12/2018 usando-se os descritores "alopecia areata and treatment" e "alopecia androgenetic and treatment". Foram utilizados filtros a fim de selecionar artigos científicos completos e gratuitos, publicados entre o período desejado e que se basearam na espécie humana.

$\mathrm{O}$ atual estudo foi efetuado em duas fases: na primeira, os artigos foram analisados pela leitura do título e resumo/abstract, ao mesmo tempo por dois revisores (BMSS e TJ), considerando os artigos de interesse. Em caso de desacordos, um terceiro revisor foi consultado (RAN). A segunda fase consistiu na leitura completa dos textos, de acordo com os critérios de inclusão e exclusão, em caso de desacordos, um último revisor foi consultado (RAN).

Para seleção dos artigos foram empregados os seguintes critérios de inclusão: artigos científicos originais presentes na base de dados PubMed publicados entre o período de 01/01/2018 a 31/12/2018, direcionados ao tratamento para alopecia areata e androgenética, disponíveis gratuitamente e baseados na espécie humana. Como critérios de exclusão foram desconsiderados os artigos científicos de revisão e os não enquadrados nos critérios de inclusão.

\section{Resultados}

A busca por "alopecia areata and treatment" utilizando-se os filtros e o período determinado resultou em 26 registros, dos quais 12 atenderam aos critérios de inclusão e 14 não atenderam. Dentre os 14 artigos excluídos, havia dois artigos de revisão, três artigos que não enfocaram no tratamento e artigos que fugiram ao tema proposto. Após a leitura dos 12 artigos na 
íntegra, apenas quatro foram utilizados por se encaixarem nos objetivos do estudo. A Figura 1 representa graficamente o esse processo de seleção e a Tabela 1 apresenta os artigos que foram utilizados na primeira busca.

Figura 1: Representação gráfica do processo de seleção dos estudos resultantes da busca por "alopecia areata and treatmnet".

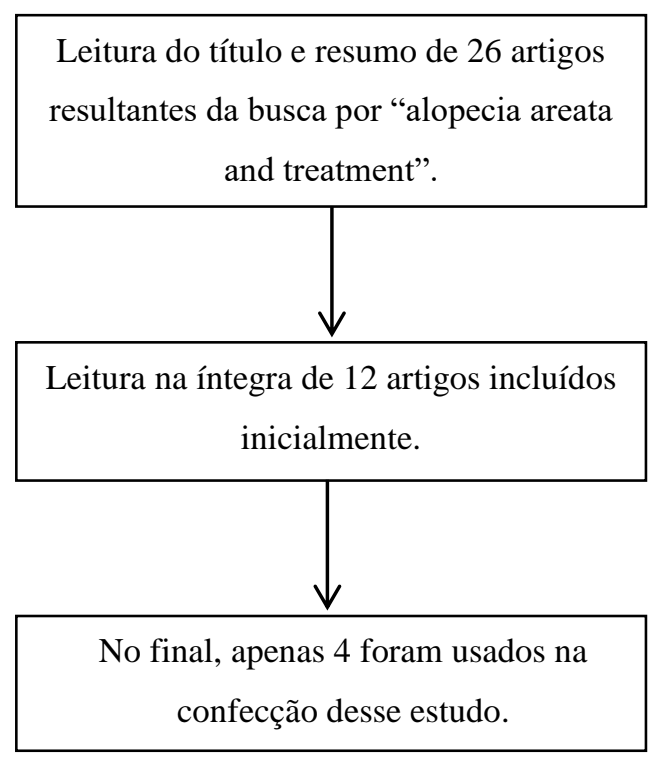

Fonte: Autores (2019).

A busca por "alopecia androgenetic and treatment" resultou em 84 registros, dos quais 24 foram incluídos e 61 artigos não atenderam aos critérios de inclusão. Após lidos os 24 artigos na íntegra, apenas nove foram utilizados na confecção desse estudo, uma vez que quatro foram excluídos por não se enquadrarem no assunto, sete por terem aparecido também na busca por "alopecia areata and treatment", um por ser artigo de revisão e três por não terem se baseado na espécie humana. A Tabela 2 retrata os artigos que foram utilizados nessa segunda busca.

Figura 2: Representação gráfica do processo de seleção dos estudos resultantes da busca por "alopecia androgenetic and treatment".

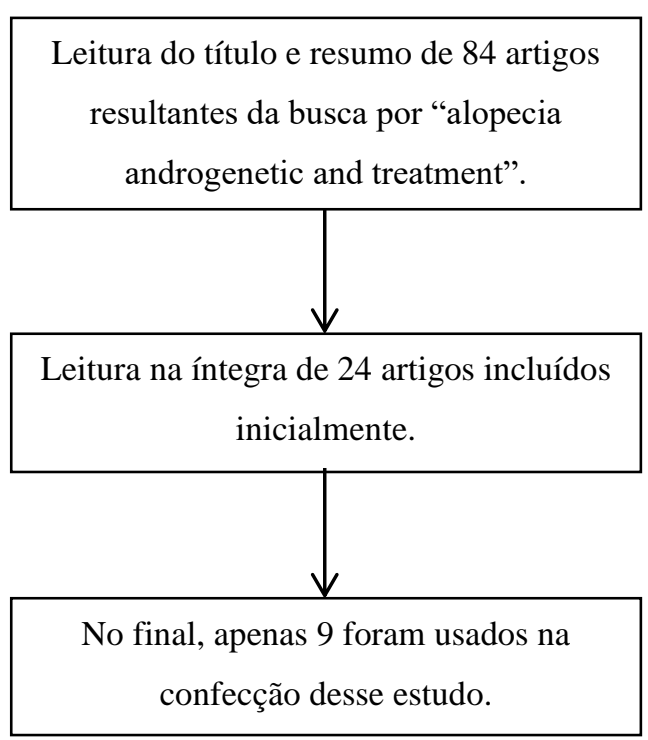

Fonte: Autores (2019). 
Após a fase de seleção, empregando-se os critérios de inclusão e exclusão previamente definidos, 13 artigos permaneceram elegíveis, sendo sete pela busca por alopecia areata e nove por alopecia androgenética. A taxa de concordância entre os dois revisores foi de 78,6\% quanto a decisão de incluir ou excluir um artigo, conforme mostram as Tabelas 01 e 02.

\subsection{Alopecia Areata}

Tabela 1: Descrição e avaliação dos estudos incluídos resultantes da busca "alopecia areata and treatment".

\begin{tabular}{|c|c|c|c|}
\hline Estudo & Tipo de estudo & $\begin{array}{l}\text { Tipo de tratamento } \\
\text { sugerido para alopecia }\end{array}$ & Resultado do estudo \\
\hline Anderi, et al., 2018 & $\begin{array}{l}\text { Ensaio clínico realizado com } 20 \\
\text { pacientes ( } 9 \text { homens e } 11 \\
\text { mulheres entre } 23 \text { e } 63 \text { anos }) .\end{array}$ & $\begin{array}{l}\text { Uso de enxerto autólogo de } \\
\text { células vasculares estromais } \\
\text { derivadas do tecido adiposo. }\end{array}$ & $\begin{array}{l}\text { Viu-se que a grande maioria dos } \\
\text { pacientes apresentou melhora na } \\
\text { densidade capilar, no diâmetro e no } \\
\text { teste de tração após uso do enxerto. }\end{array}$ \\
\hline Rodgers, 2018 & $\begin{array}{l}\text { Trata-se de uma série de casos } \\
\text { feita com membros anônimos } \\
\text { de um grupo de apoio da } \\
\text { Função Nacional de Alopecia } \\
\text { Areata da Califórnia (NAAF). }\end{array}$ & $\begin{array}{l}\text { Tratamento multidisciplinar } \\
\text { completor incluindo } \\
\text { tratamento farmacológico e da } \\
\text { saúde mental. }\end{array}$ & $\begin{array}{l}\text { Concluiu-se a importância de aliar o } \\
\text { tratamento farmacológico (para } \\
\text { controlar a doença e passar ao paciente } \\
\text { uma sensação de controle sobre o } \\
\text { corpo) com tratamento da saúde mental } \\
\text { (essa pode complicar a doença } \\
\text { dermatológica e comprometer a } \\
\text { autoestima). }\end{array}$ \\
\hline Hamed, et al., 2018 & $\begin{array}{l}\text { Imunoensaio clínico feito } \\
\text { extraindo-se células HaCat in } \\
\text { vrito, extraídas de pacientes } \\
\text { com alopecia areata, as quais } \\
\text { foram incubadas com } \\
\text { interferon-gama e tratadas com } \\
\text { epigalocatequina-3-galato em } \\
20 \text { ou } 40 \text { micrômetros por } 24 \mathrm{ou} \\
48 \text { horas. }\end{array}$ & $\begin{array}{l}\text { Uso de epigalocatequina-3- } \\
\text { galato como candidato ao } \\
\text { tratamento da alopecia areata } \\
\text { ao invés dos inibidores da } \\
\text { JAK. }\end{array}$ & $\begin{array}{l}\text { Houve redução na expressão de genes } \\
\text { da via do interferon-gama, como HLA- } \\
\text { B, HLA-BR, IRF-1 e STAT-1. Viu-se } \\
\text { que o epigalocatequina-3-galato é mais } \\
\text { seguro e mais específico que os } \\
\text { inibidores da JAK. }\end{array}$ \\
\hline $\begin{array}{l}\text { Pekmezci, et al., } \\
2018 .\end{array}$ & $\begin{array}{l}\text { Imunoensaio clínico feito com } \\
\text { cultura de células HaCat in vitro } \\
\text { a partir de pele } \\
\text { histologicamente normal. } \\
\text { Nessas células foi usado um } \\
\text { conjunto de extratos herbais. }\end{array}$ & $\begin{array}{l}\text { Composição para uso tópico } \\
\text { no tratamento da alopecia não } \\
\text { cicatricial, constituída de seis } \\
\text { extratos herbais. }\end{array}$ & $\begin{array}{l}\text { Nas células HaCat, houve inibição da } \\
\text { interleucina-1-alfa, um } \\
\text { antiproliferativo. Além disso, os } \\
\text { extratos possuem diversas propriedades } \\
\text { benéficas: ação anti-inflamatória, } \\
\text { antioxidante, estímulo da angiogênese, } \\
\text { ação do silício nos folículos pilosos, } \\
\text { gerando fios mais grossos e outros } \\
\text { efeitos. }\end{array}$ \\
\hline
\end{tabular}

Fonte: Autores (2019).

Anderi et al. (2018) apontaram outra forma de tratamento de alopecia areata, usando-se enxerto autólogo de células vasculares estromais derivadas do tecido adiposo. O estudo analisou 20 pacientes, sendo nove mulheres e 11 homens, todos com idade entre 23 e 63 anos, no Oriente Médio, diagnosticados com alopecia areata e que receberam o enxerto no couro cabeludo. Como resultado, 19 dos 20 pacientes apresentaram melhora no diâmetro do cabelo, obtendo uma média de $32 \%$ de melhora. Quanto à densidade capilar, 18 dos 20 pacientes apresentaram melhora, sendo que o crescimento médio foi de $36 \%$. Outra melhora significativa obtida foi quanto ao teste de tração (teste da puxada), em que 18 dos 20 pacientes apresentaram melhora, com diminuição significativa no número de cabelos extraídos (Anderi, et al., 2018).

Rodgers (2018) assinalou a importância de um tratamento da alopecia areata completo multidisciplinar que incluísse saúde mental e farmacologia a partir de entrevistas com membros anônimos de um grupo de apoio da Função Nacional de Alopecia Areata da Califórnia (NAAF). O artigo aponta que não abordar a saúde mental pode complicar a doença dermatológica em si, a gestão farmacológica da doença, os relacionamentos interpessoais, a qualidade de vida do paciente e a 
autoestima, podendo gerar pensamentos suicidas. Podem ser oferecidas como medidas para lidar com tais questões: grupos de apoio, técnicas de relaxamento e reflexão. Além do tratamento focado na saúde mental, também é de extrema importância instaurar um tratamento farmacológico, uma vez que foi visto em um grupo de apoio de membros anônimos da Califórnia que encontrar um medicamento para a doença aumentaria o senso de confiança, autoestima e controle sobre o corpo (Rodgers, 2018).

Hamed et al. (2018) observaram os efeitos do epigalocatequina-3-galato na resposta inflamatória da alopecia areata. Como método, foram escolhidos 20 pacientes com alopecia areata do departamento de Dermatologia do Royal Hallamshire Hospital no Reino Unido. Foram usadas células HaCat in vitro (linhas de queratinócitos humanos), as quais foram incubadas com interferon-gama para induzir a fosforilação da proteína de transcrição STAT-1 e em seguida tratadas com epigalocatequina-3-galato em 20 ou 40 micrômetros por 24 ou 48 horas (Hamed, et al., 2018).

Houve uma diminuição de STAT-1 em 35\% em células tratadas com 20 micrômetros, e diminuição em $81 \%$ quando usados 40 micrômetros. Células $\mathrm{HaCat}$ quando tratadas com 40 micrômetros de epigalocatequina-3-galato por 48 horas manifestaram, além da expressão diminuída de STAT-1, inibição significativa da JAK-2, mas a expressão de JAK-1 não foi afetada. O estudo verificou também a expressão das moléculas HLA-DR e HLA-B e viu-se que os níveis de HLA-DR não se alteraram quando usados 20 micrômetros de epigalocatequina-3-galato após 48 horas, mas tiveram significativas reduções com 40 micrômetros. No entanto, foi observada uma significativa redução de HLA-B em doses de 20 e mantidas com 40 (Hamed, et al., 2018).

Como conclusão, o artigo propõe o epigalocatequina-3-galato como candidato ao tratamento da alopecia areata ao invés dos inibidores da JAK (como ruxolitinib, tofacitinib e baricitini), uma vez que estes têm custos relativamente altos e muitos efeitos colaterais. O epigalocatequina-3-galato tem propriedades anti-inflamatórias por ser capaz de reduzir a expressão de genes da via do interferon-gama, como HLA-B, HLA-BR, IRF-1 e STAT-1, via que age na patogênese da alopecia areata e se inibida pode representar uma forma de terapia. Além disso, o epigalocatequina-3-galato inibe especificamente a via de expressão JAK-2 e não a JAK-1, o que não reduz sua eficiência e lhe mais seguranças que os inibidores da JAK (Hamed, et al., 2018).

Pekmezci, Dundar e Turkoglu (2018) formularam uma composição para uso tópico constituída de seis extratos herbais com ação anti-inflamatória e antioxidante para alopecia não cicatricial. As ervas usadas foram: extrato de raiz de Urtica dioica, extrato de folha de Urtica urens, extrato de folha do Equisetum arvense, extrato da parte aérea de Achillea millefolium, extrato da folha de Matricaria chamomilla e extrato do fruto de Ceratonia siliqua. O extrato foi usado em cultura de células HaCat in vitro retiradas da pele histologicamente normal, essas foram submetidas a diferentes concentrações da solução de ervas (Pekmezci, Dundar \& Turkoglu, 2018).

O resultado mais importante foi a inibição da interleucina-1-alfa nas células, a qual age diretamente nos folículos pilosos inibindo o crescimento, é um antiproliferativo de crescimento capilar. Além desse fator, outras vantagens do extrato herbal são as diversas propriedades das ervas utilizadas, a Urtica dioica contém sitosterol beta, que estimula a angiogênese, aumentando a síntese do fator de crescimento endotelial vascular e suporta o crescimento de novos fios. A Urtica urens tem propriedades anti-inflamatórias e antioxidantes, agindo no mecanismo patogênico da alopecia. O Equisetum arvense é um acumulador de silício, que penetra nos folículos pilosos e permite que os fios cresçam mais grossos, fato esse que também resulta na diminuição da queda. A Achillea millefolium tem propriedades anti-inflamatórias, a Matricaria chamomilla estimula a fase catágena no ciclo de crescimento capilar (fase de transição entre a fase anágena e telógena, em que o fio ainda não se destacou do couro cabeludo) e a Ceratonia siliqua tem propriedades anti-oxidantes. Devido a todos esses benefícios citados, o extrato herbal em questão pode ser considerado um agente promissor no tratamento da alopecia (Pekmezci, Dundar \& Turkoglu, 2018). 


\subsection{Alopecia Androgenética}

Tabela 2: Descrição e avaliação dos estudos incluídos resultantes da busca "alopecia androgenetic and treatment".

\section{Estudo}

Tipo de estudo

\section{Tipo de tratamento sugerido} para alopecia

\section{Resultado do estudo}

Zhang, et al., 2018 Imunoensaio clínico realizado com Uso da finasterida e avaliação

178 pacientes com alopecia dos níveis séricos de
androgenética e 61 controles sem a andrógenos após o tratamento.
doença.
doença.

Viu-se que os níveis séricos de testosterona total, testosterona livre e di-hidrotestosterona andrógeno foram menores que os níveis prétratamento. Não houve diferença significativa entre os níveis de globulina ligadora de hormônios sexuais. Viu-se também que os níveis séricos diminuídos de dihidrotestosterona andrógeno podem ser benéficos, desde que não reduzidos excessivamente.

Wang, et al., 2018 Estudo transversal feito com 355 Tratamento voltado para a Alunos com alopecia androgenética estudantes universitários de 18 abordagem psicológica, além universidades do sul da China com do tratamento da queda de aloepcia areata e 406 controles sem a cabelo. doença.

\begin{tabular}{|c|c|c|}
\hline $\begin{array}{l}\text { Faghihi, et al., } \\
2018\end{array}$ & $\begin{array}{l}\text { Ensaio clínico realizado com grupo } \\
\text { caso (recebeu Minoxidil a } 5 \% \text { mais } \\
\text { terapia de luz de baixo nível) e } \\
\text { controle (recebeu terapia de pente a } \\
\text { laser, que foi desligada para agir } \\
\text { como placebo, e Minoxidil da mesma } \\
\text { maneira que o grupo caso). }\end{array}$ & $\begin{array}{l}\text { Minoxidil a } 5 \% \text { associada } \\
\text { terapia de luz de baixo nível. }\end{array}$ \\
\hline $\begin{array}{l}\text { Kaliyadan,et al., } \\
2018\end{array}$ & Relato de caso & $\begin{array}{l}\text { Aplicação intralesional de } \\
\text { acetonido de triancinolona em } \\
\text { dispositivo de injeção sem } \\
\text { agulha, associada à fototerapia. }\end{array}$ \\
\hline $\begin{array}{l}\text { Hawkshaw,et al., } \\
2018\end{array}$ & & $\begin{array}{lrr}\text { Uso de } & \text { WAY-316606, } & \text { um } \\
\text { antagonista } & \text { específico } & \text { de } \\
\text { SFRP1. } & & \end{array}$ \\
\hline
\end{tabular}
apresentaram mais agressividade, dificuldade interpessoal, depressão, somatização, comportamento obsessivo-compulsivo e ansiedade que os controles, principalmente alunos de medicina (submetidos a muito estresse) e de arte (expõem-se muito ao público).

Houve maior recuperação da alopecia no grupo caso, bem como maior satisfação pessoal, demonstrando a importância de aliar o minoxidil com a terapia de luz de baixo nível.

Houve despigmentação e atrofia nas áreas de aplicação, um efeito colateral comum. Um achado incomum foi o crescimento de pelos significativo ao redor dessas áreas atróficas, muito maior do que ao redor das áreas sem atrofia. cabeludo humano com ciclosporina A, ex vivo, havendo a inibição do Wnt pela proteína 1 secretada frisada (SFRP1). Com base nisso, foi usado o WAY-316606 por 6 dias, um antagonista específico de SFRP1, o qual promoveu o crescimento capilar ex vivo.

\begin{tabular}{lll}
\hline Tsai, et al., 2018 & $\begin{array}{l}\text { Estudo clínico randomizado com 117 } \\
\text { homens heterossexuais com alopecia } \\
\text { androgenética. }\end{array}$ & $\begin{array}{l}\text { Uso da dutasterida e análise dos } \\
\text { seus efeitos adversos sexuais. }\end{array}$ \\
&
\end{tabular}
20 pacientes apresentaram tais eventos: 9 eram do grupo de tratamento duplo-cego com dutasterida, 5 do grupo de tratamento ativo com finasterida, 5 do grupo que recebeu placebo e um após o placebo ser descontinuado. Nenhum evento adverso persistiu após a interrupção do medicamento.

\begin{tabular}{|c|c|c|c|}
\hline Arita, et al., 2018 & Relato de caso. & $\begin{array}{l}\text { Tratar a dermatite atópica, uma } \\
\text { vez que a disposição atópica e a } \\
\text { doença autoimune podem } \\
\text { predispor à alopecia areata. }\end{array}$ & $\begin{array}{l}\text { A remissão completa da alopecia areta de uma } \\
\text { paciente de } 57 \text { anos de idade com dermatite } \\
\text { atópica aconteceu após a paciente abandonar } \\
\text { o hábito de dormir com seu cachorro. }\end{array}$ \\
\hline Ganzer, et al., 2018 & $\begin{array}{l}\text { Estudo transversal feito com } \\
\text { aplicação de questionário online com } \\
97 \text { homens em uso de } 1 \mathrm{mg} \text { de } \\
\text { finasterida por mais de } 3 \text { meses para } \\
\text { tratar alopecia androgenética. }\end{array}$ & $\begin{array}{l}\text { Uso da finasterida de forma } \\
\text { consciente, pesquisando-se o } \\
\text { histórico psiquiátrico dos } \\
\text { pacientes com alopecia } \\
\text { androgenética antes de } \\
\text { prescreve-la. }\end{array}$ & $\begin{array}{l}\text { Viu-se que mais da metade dos pacientes que } \\
\text { relataram "síndrome pós-finasterida", isso é, } \\
\text { medo, desesperança e ansiedade, já tinham } \\
\text { diagnósticos psiquiátricos pregressos de } \\
\text { ansiedade, depressão e outros. }\end{array}$ \\
\hline Tenore, et al., 2018 & $\begin{array}{l}\text { Ensaio clínico realizado com } 250 \\
\text { pacientes com calvície. } 125 \\
\text { receberam o extrato polifenólico da } \\
\text { mação Annurca microencapsulado } \\
\text { com maltodextrinas e } 125 \text { receberam } \\
\text { essa mesma composição acrescida de } \\
\text { biotina, selenometionina e acetato de } \\
\text { zinco, denominado AppleMets Hair. }\end{array}$ & $\begin{array}{l}\text { Uso da procianidina } \mathrm{B} 2 \text {, um } \\
\text { extrato polifenólico da maçã } \\
\text { Annurca, nativa do sul da Itália. }\end{array}$ & $\begin{array}{l}\mathrm{O} \text { grupo que recebeu o AppleMets Hair teve } \\
\text { maior número de cabelo/ } \mathrm{cm}^{2} \text {, maior aumento } \\
\text { no peso do cabelo e maior teor da queratina. }\end{array}$ \\
\hline
\end{tabular}


Zhang et al. (2018) avaliaram os efeitos antiandrogênicos das drogas utilizadas no tratamento da alopecia androgenética, como distúrbios gastrointestinais e hepáticos, mastalgia, hipossexualidade e disfunção erétil. Tais efeitos podem impactar no estado psicológico do paciente e, por isso, devem ser levados em conta ao escolher a droga utilizada (Zhang, et al., 2018).

O estudo analisou 178 pacientes masculinos entre 20 e 40 anos de um hospital universitário de Zhejiang com alopecia androgenética e 61 controles sem a doença com o objetivo de medir os níveis séricos de andrógeno antes e depois do tratamento com finasterida. Foram medidos os níveis séricos de testosterona, testosterona livre e globulina ligadora de hormônios sexuais por imunoensaio de quimioluminscência e di-hidrotestosterona andrógeno por radioimunoensaio (Zhang, et al., 2018).

Os níveis pré-tratamento de testosterona total, a testosterona livre e di-hidrotestosterona andrógeno foram maiores nos indivíduos com alopecia, enquanto os níveis de globulina ligadora de hormônios sexuais foram semelhantes nos grupos com alopecia e nos controles normais. Após a terapia anti-androgênica, viu-se que os níveis séricos de testosterona total, testosterona livre e di-hidrotestosterona andrógeno foram menores que os níveis pré-tratamento. Não houve diferença significativa entre os níveis de globulina ligadora de hormônios sexuais antes e após a terapia. 136 pacientes tiveram níveis séricos de di-hidrotestosterona andrógeno diminuídos em relação ao pré-tratamento, nesses, houve uma taxa curativa maior. O estudo viu que essa diminuição pré e pós tratamento teve resultados melhores na terapia contra alopecia se a diminuição percentual entre pré e pós uso de finasterida for menor que 50\%, e resultados piores se a diminuição percentual for maior que 50\% (Zhang, et al., 2018).

Logo, os níveis séricos diminuídos de di-hidrotestosterona andrógeno podem ser benéficos para tratar a alopecia androgenética, mas podem ser prejudiciais se diminuírem excessivamente. Além desses resultados, verificou-se também ligeiro aumento nos níveis de LH e FSH após a terapia antiandrogênica em 55 pacientes e esse aumento apresentou-se relacionado a uma menor taxa curativa. Pode-se concluir desse estudo que a finasterida reduz os níveis de di-hidrotestosterona, contudo, deve-se atentar para que essa diminuição não seja excessiva (Zhang, et al., 2018).

Wang et al. (2018) buscaram avaliar o estado psicológico de 355 estudantes universitários de 18 universidades do sul da China com alopecia androgenética e de 406 controles saudáveis, entre 17 e 40 anos. Os resultados mostraram que o grupo de pessoas com alopecia androgenética apresentou mais agressividade, dificuldade interpessoal, depressão, somatização, comportamento obsessivo-compulsivo e ansiedade quando comparado ao grupo de controles. Viu-se que a alopecia pode exercer pressão maior nos estudantes de arte, uma vez que esses estão mais expostos ao público, e nos estudantes de medicina, pois esses naturalmente estão mais suscetíveis a depressão e sensibilidade interpessoal, mesmo sem a alopecia. Logo, viu-se que o tratamento da alopecia androgenética deve conter abordagem psicológica, principalmente em pacientes universitários (Wang, et al., 2018).

Outra terapia disponível para o tratamento da alopecia androgenética é a terapia com luz de baixo nível à solução de Minoxidil a 5\%, proposta por Faghihi et al. (2018). Essa terapia estimula a reentrada dos folículos capilares telógenos na fase anágena, prolonga a fase anágena, retarda a catágena e estimula a proliferação dos folículos pilosos (Faghihi, et al., 2018).

O estudo foi realizado em 2014 e 2015 com pacientes de 17 a 45 anos de idade, o grupo caso recebeu 20 gotas de Minoxidil a 5\% duas vezes ao dia por 6 meses, mais duas a três sessões de 20 minutos de terapia de luz de baixo nível por 6 meses. O grupo controle recebeu terapia de pente a laser, que foi desligada para agir como placebo, e Minoxidil da mesma maneira que o grupo caso (Faghihi, et al., 2018).

Houve maior recuperação da alopecia no grupo caso, bem como maior satisfação pessoal. A contagem de cabelo do grupo caso foi maior em relação ao controle principalmente no nono e no décimo segundo mês, o diâmetro capilar foi maior apenas a partir do décimo segundo mês. Logo, o Minoxidil associado à terapia de luz baixa pode aumentar a recuperação da 
alopecia androgenética e a satisfação dos pacientes (Faghihi, et al., 2018).

Kaliyadan, Alkhateeb e Swaroop (2018) demostraram em um estudo os efeitos da aplicação intralesional de acetonido de triancinolona em dispositivo de injeção sem agulha, associada à fototerapia. Houve despigmentação e atrofia nas áreas de aplicação, um efeito colateral comum das injeções. Um achado incomum foi o crescimento de pelos significativo ao redor dessas áreas atróficas, muito maior do que ao redor das áreas sem atrofia (Kaliyadan, Alkhateeb \& Swaroop, 2018).

Atualmente, há apenas dois medicamentos aprovados pela Food And Drug Administration's (FDA) para o tratamento da perda de cabelo: a finasterida e o Minoxidil, os quais são insatisfatórios por terem resultados variáveis entre os indivíduos e pelos seus efeitos colaterais. Um dos estudos, realizado por Hawkshaw (2018), buscou tratar folículos capilares do couro cabeludo humano com ciclosporina $\mathrm{A}$, essa induz a fase anágena em folículos capilares telógenos e inibe a regressão de folículos capilares catágenos (Hawkshaw, 2018).

Os folículos foram obtidos do couro cabeludo occipital masculino de pacientes submetidos a transplante capilar, foram cultivados e incubados com ciclosporina A. Após essa experiência, verificou-se a inibição do Wnt (age na via de sinalização em renovação e proliferação celular) pela proteína 1 secretada frisada (SFRP1). Com base nisso, foi usado o WAY316606 por 6 dias, um antagonista específico de SFRP1, muito bem tolerado, o qual promoveu o crescimento capilar ex vivo. Houve aumento significativo do alongamento da haste capilar 2 dias após o tratamento com o WAY-316606, mais rápido do que o alongamento produzido pela ciclosporina A, que leva mais dias, houve também maior expressão proteica da queratina K85 da haste capilar (Hawkshaw, 2018).

Tsai et al. (2018) pesquisaram os efeitos sexuais do dutasterida no tratamento da alopecia androgenética. Esse é um inibidor da 5-alfa-redutase, bem como a finasterida, e pode causar efeitos colaterais, como a redução da libido e disfunção erétil. Os 117 participantes do estudo eram homens heterossexuais entre 23 e 50 anos com relacionamento estável há mais de 6 meses. Foram 4 semanas de placebo cego e tratamento duplo-cego por 24 semanas recebendo-se $0,5 \mathrm{mg}$ de dutasterida ou placebo. Depois, os participantes foram tratados ativamente com $0,5 \mathrm{mg}$ de dutasterida por 24 semanas, seguidas de quatro semanas de acompanhamento pós-tratamento (Tsai, et al., 2018).

Ocorreram efeitos adversos em um terço dos pacientes, sendo que $31 \%$ dos efeitos ocorreram nos pacientes que receberam o placebo, $33 \%$ nos que receberam a dutasterida e $29 \%$ nos que tiveram tratamento ativo com dutasterida por 24 semanas. Aproximadamente metade dos pacientes que foram tratados com o medicamento por mais de 48 semanas relataram pelo menos um evento adverso. Durante a fase de tratamento duplo-cego, a incidência de eventos adversos sexuais foi duas vezes maior no grupo que recebeu dutasterida $(16 \%)$ do que no grupo placebo $(8 \%)$, mas menor $(5 \%)$ que no grupo de tratamento ativo com dutasterida. A maioria dos eventos adversos sexuais começaram nos primeiros 3 meses de tratamento. Dos 20 pacientes que apresentaram tais eventos, 9 eram do grupo de tratamento duplo-cego com dutasterida, 5 do grupo de tratamento ativo, 5 do grupo que recebeu placebo e um após o placebo ser descontinuado. Todos os eventos adversos sexuais foram tolerados, esperados, apareceram precocemente, se resolveram durante o tratamento e nenhum persistiu após a interrupção do uso da dutasterida (Tsai, et al., 2018).

Arita et al. (2018) comprovaram que possuir história de dermatite atópica pode atuar como gatilho para o aparecimento da alopecia areata. No estudo, a paciente de 57 anos de idade com dermatite atópica leve buscou tratamento para perda de cabelo, que se iniciou no couro cabeludo e se espalhou para o resto do corpo. A paciente foi tratada com corticosteroide tópico, anti-histamínico e cefantantina por um ano, crioterapia por 14 meses e terapia de luz de excimer em intervalos de duas semanas em 24 sessões. O crescimento capilar foi parcialmente alcançado, mas a alopecia recorreu, foi quando se desconfiou da dermatite atópica, uma vez que a paciente dormia com seu cachorro há 10 anos e seus níveis de IgE específicos contra pelos de cachorros eram muito altos (2460 UA/mL) (Arita, et al., 2018).

Foi recomendando que a paciente não dormisse com seu cachorro, e após três meses de acompanhamento houve 
melhora gradual da alopecia, havendo remissão completa em dois anos e sete meses, além disso, a dermatite atópica melhorou e os níveis de IgE se mostraram inferiores (Arita, et al., 2018).

Um estudo realizado por Ganzer e Jacobs (2018) buscou avaliar os efeitos físicos e psicológicos obtidos após o uso da finasterida, também chamados de "síndrome pós-finasterida". A avaliação foi feita através de um questionário online, em 97 participantes do sexo masculino, com mais de 18 anos, com alopecia androgenética e em uso de $1 \mathrm{mg}$ de finasterida por mais de três meses (Ganzer \& Jacobs, 2018).

Os resultados desse estudo foram: 87 participantes (89,7\%) relataram estar em boa saúde e 53 participantes (55\%) tinham algum diagnóstico psiquiátrico estabelecido antes de iniciar a terapia com finasterida, como a depressão e ansiedade, o que coloca o paciente em risco de desenvolver alterações emocionais pós-finasterida. Havia uma pergunta aberta para avaliar qualitativamente a experiência de ter tomado a finasterida e as respostas envolviam "desespero", "medo", "falta de esperança", "a pior coisa que já aconteceu", "custou todo o dinheiro que tinha e arruinou o relacionamento emocional pela vida". Isso mostra a importância de os médicos prescritores investigarem o histórico psiquiátrico dos seus pacientes antes de prescrever a finasterida para o tratamento da alopecia androgenética (Ganzer \& Jacobs, 2018).

Tenore et al. (2018) investigaram os efeitos do extrato polifenólico da maçã Annurca, nativa do sul da Itália. Foram feitos suplementos em formato de cápsulas preparados de duas maneiras: um rico em extrato polifenólico da maça Annurca microencapsulado com maltodextrinas ( $400 \mathrm{mg}$ ) e outro rico em extrato polifenólico da maça Annurca microencapsulado com maltodextrinas, biotina $(0,20 \mathrm{mg})$, selenometionina (80,0 microgramas) e acetato de zinco $(21,0 \mathrm{mg})$, esse segundo tipo denominado "AppleMets Hair" (Tenore, et al., 2018).

Os participantes do estudo tinham entre 30 e 83 anos com evidências de calvície, o estudo durou 16 semanas, sendo 4 semanas de tratamento com placebo, 8 semanas de tratamento recebendo o composto formulado e 4 semanas de acompanhamento. 250 pacientes foram aleatoriamente distribuídos para receberem o suplemento rico apenas no extrato da maçã Annurca ou para receber o AppleMets Hair, ficando 125 pacientes em cada grupo, os pacientes tomaram duas cápsulas por dia (uma de manhã e outra à noite) (Tenore, et al., 2018).

Como resultados, em ambos os grupos, não houve diferença de número de cabelo, peso e teor da queratina ao final do período de uso do placebo. Contudo, houve diferença entre os grupos ao final do tratamento com o suplemento, sendo que os resultados foram mais significativos no grupo que recebeu AppleMets Hair, havendo aumento no número de cabelo/ $/ \mathrm{cm}^{2} \mathrm{em}$ $125,2 \%$ (enquanto o do outro grupo foi de $118,3 \%$ ), aumento do peso do cabelo em $42,1 \%$ (do outro grupo foi de $37,3 \%$ ) e aumento do teor da queratina em 40,1\% (o do outro grupo foi de 35,7\%) (Tenore, et al., 2018).

Logo, conclui-se que além da procianidinas oligoméricas serem capazes de promover o crescimento de células epiteliais capilares, viu-se que a coformulação do suplemento usando-se a procianidina B2 extraída da maçã Annurca junto com a vitamina biotina, zinco e seleno levou a resultados ligeiramente superiores em termos de número do cabelo/ $\mathrm{cm}^{2}$, peso do cabelo e teor da queratina, indicando claramente o papel desses elementos no crescimento capilar (Tenore, et al., 2018).

\section{Discussão}

Os estudos abordados demonstraram a vasta gama de tratamentos disponíveis para a alopecia areata e a androgenética, demonstrando a importância de direcionar a terapêutica para uma abordagem individual. Alguns estudos analisaram os inibidores da 5-alfa-redutase, como a dutasterida, a qual possui eventos adversos tolerados, esperados e autolimitados; e a finasterida, a qual reduz os níveis de andrógeno ao longo do tratamento, porém essa redução é benéfica para a terapêutica, desde que não seja de forma excessiva. Além disso, devido aos eventos adversos que podem ocorrer (síndrome pósfinasterida), é fundamental pesquisar o histórico psiquiátrico do paciente antes de receitar o medicamento, pois diagnósticos psiquiátricos pregressos podem intensificar essa síndrome. Outro diagnóstico pregresso que deve ser pesquisado é o de 
dermatite atópica, uma vez que essa pode interferir no tratamento, devido ao seu mecanismo autoimune que age na via patogênica da alopecia areata.

O minoxidil, um hipotensor, também é um medicamento promissor para o tratamento da alopecia, contudo, ele é mais eficaz se associado à terapia de luz de baixo nível em comparação ao seu uso isolado, logo, essa associação deve ser levada em conta ao prescreve-lo para o paciente.

Também foram abordadas nos estudos, extratos herabis e suas ricas propriedades que permitem o crescimento capilar, como o extrato polifenólico da maçã Annurca, extrato de raiz de Urtica dioica, extrato de folha de Urtica urens, extrato de folha do Equisetum arvense, extrato da parte aérea de Achillea millefolium, extrato da folha de Matricaria chamomilla e extrato do fruto de Ceratonia siliqua.

Um ponto extremamente relevante de alguns estudos foi a importância da abordagem multidisciplinar, uma vez que a parte psicológica muitas vezes é abandonada pelos profissionais da saúde. Essa ajuda o paciente a melhorar sua autoestima, a qual está abalada na alopecia pelo fato de o cabelo ser um instrumento de comunicação psicossocial.

Os estudos também trouxeram outros tratamentos inovadores, tais como o enxerto autólogo de células vasculares estromais derivadas do tecido adiposo, uso do WAY-316606, a aplicação intralesional de acetonido de triancinolona com fototerapia e o uso de epigalocatequina-3-galato, a qual demonstrou ser mais segura que os inibidores da JAK (ruxolitinib e tofacitinib). No total, tais estudos em questão demonstraram sucesso e eficácia.

\section{Conclusão}

Concluindo, deve-se estimular a confecção de mais estudos sobre este tema, uma vez que, com os diversos tratamentos disponíveis para a alopecia, é necessário estudá-los e analisá-los detalhadamente, verificando seus benefícios e malefícios.

Além disso, vê-se a necessidade de difundir a ideia de aliar o tratamento farmacológico com o tratamento psicológico do paciente, sendo que este último é muitas vezes deixado de lado, comprometendo o resultado da terapia farmacológica e afetando a saúde mental do paciente.

Durante o tratamento, vê-se também a necessidade de pesquisar o histórico do paciente e suas doenças pregressas, uma vez que doenças psiquiátricas podem contribuir para a redução da autoestima e doenças atópicas autoimunes podem atuar como gatilho para a alopecia.

\section{Referências}

Anderi, R., Makdissy, N., Azar, A., Rizk, F., \& Hamade, A. (2018). Cellular therapy with human autologous adipose-derived adult cells of stromal vascular fraction for alopecia areata. Stem Cell Research \& Therapy. 10.1186/s13287-018-0889-y

Arita, T., Nomiyama, T., Asai, J., \& Katoh, N. (2018). Successful treatment of refractory alopecia universalis by persuading a patient not to sleep with her dog. Allergology International. 10.1016/j.alit.2017.06.011

Carvalho, L. T., \& D’Acri, A. M. (2014). Alopecia Areata: Revisão Bibliográfica e Relato de Caso. Cad. Bras. Med. XXVII. http://www.seer.unirio.br/index.php/cadernosbrasileirosdemedicina/article/view/4422/3983

Darwin, E. (2018). Alopecia Areata: Review of Epidemiology, Clinical Features, Pathogenesis and New Treatment Options. Int. Jornaul of Trichology. 10.4103/ijt.ijt_99_17

Dawber, R., \& Van Neste, D. (1996). Doenças dos Cabelos e do Couro Cabelo. Ed. Manole. p. 205-7

Dhaher, S. A., \& Yacoub, A. A. (2018). Estimation of Zinc and Iron Levels in the Serum and Hair of Women with Androgenetic Alopecia: Case-control Study. Indian Journal of Dermatology. 10.4103/ijd.IJD_624_16

Ejjiyar, M., \& Driss El Amrani, M. (2018). An unusual cause of alopecia on the scalp. The Pan African Medical Journal. 10.11604/pamj.2018.29.213.14533 
Faghihi, G. (2018). The effectiveness of adding low-level light therapy to monoxidil 5\% solution in the treatment of patients with androgenetic alopecia. Indian J. Dermatol Venereol Leprol. 10.4103/ijdvl.IJDVL_1156_16

Faghihi, G., Mozafarpoor, S., Asilian, A., Mokhtari, F., Esfahani, A. A., Bafandeh, B., Nouraei, S., Nilforoushzadeh, M. A., \& Hosseini, S. M. (2018). The effectiveness of adding low-level light therapy to minoxidil $5 \%$ solution in the treatment of patients with androgenetic alopecia. Indian $J$ Dermatol Venereol Leprol. 10.4103/ijdvl.IJDVL_1156_16

Ganzer, C. A., \& Jacobs, A. R. (2018). Emotional Consequences of Finasteride: Fool's Gold. American Journal of Men's Health. 10.1177/1557988316631624

Gomez-Zubiaur, A., Saceda-Corralo, D., Velez-Velazquez, M. D., \& Vano-Galvan, S. (2018). Tratamiento de la alopecia areata, um recorrido desde las opciones terapêuticas clásicas hasta los nuervos fármacos aparecidos em los últimos años. Dermatology Online Journal. https://escholarship.org/uc/item/4ft667kk

Gonul, M., Cemil, B. C., Ayvaz, H. H., Cankurtaran, E., Ergin, C., \& Gurel, M. S. (2018). Comparision of quality of life in patients with androgenetic alopecia and alopecia areata. An. Bras. Dermatol. 10.1590/abd1806-4841.20186131

Hamed, F. N., McDonagh, A. J. G., Almaghrabi, S., Bakri, Y., Messenger, A.G., \& Tazi-Ahnini, R. (2018). Epigallocatechin-3 Gallate Inhibits STAT1/JAK2/IRF-1/HLA-DR/HLA-B and Reduces CD8 MKG2D Lymphocytes of Alopecia Areata Patient. International Journal of Environmental Research and Public Health. 10.3390/ijerph15122882

Hawkshaw, N. J. (2018). Identifying novel strategies for treating human hair loss disorders: Cyclosporine A suppresses the Wnt inhibitor, SFRP1, in the dermal papilla of human scalp hair follicles. PLOS Biology. 10.1371/journal.pbio.2003705

Kaliyadan, F., Alkhateeb, A., \& Swaroop, K. (2018). Localized atrophy with surrounding hair growth in a case of alopecia areata treated with intralesional steroids, using a needle-less injector. Indian J Dermatol Venereol Leprol. 10.4103/ijdvl.IJDVL_297_17

Martinez-Jacobo, L., Villarreal-Villarreal, C. D., Ortiz-López, R. Ocampo-Candiani, J., \& Rojas-Martínez, A. (2018). Genetic and molecular aspects of adrogenetic alopecia. Indian J Dermatol Venerol Leprol. 10.4103/ijdvl.IJDVL_262_17

Oliveira, I., \& Machado, C. C. (2017). Calvície e alopecia: Revisão Bibliográfica. Universidade Lusófona de Humanidade e Tecnologias. Recuperado de http://recil.grupolusofona.pt/bitstream/handle/10437/8402/Calv\%C3\%ADcieeAlopecia_Revis\%C3\%A3oBibliografica.pdf?sequence=1

Pekmezci, E., Dundar, C., \& Turkoglu, M. (2018). Proprietary Herbal Extract Downregulates the Gene Expression of IL-1 $\alpha$ in HaCaT Cells: Possible Implications Against Nonscarring Alopecia. Med Arch. 10.5455/medarh.2018.72.136-140

Rodgers, A. R. (2018). Why finding a treatment for alopecia areata is important: a multifaceted perspective. Journal of Investigative Dermatology Symposium Proceedings. 10.1016/j.jisp.2017.10.008

Tenore, G. C., Caruso, D., Buonomo, G., D'Avino, M., Santamaria, R., Irace, C., Piccolo, M., Maisto, M., \& Novellino, E. (2018). Annurca Apple Nutraceutical Formulation Enhances Keratin Expression in a Human Model of Skin and Promotes Hair Growth and Tropismin a Randomized Clinical Trial. Journal of Medicinal Food. 10.1089/jmf.2017.0016

Thanomkitti, K., Kanlaya, R., Fong-ngern, K., Kapincharanon, C., Sueksakit, K., Chanchaem, P., Thuangtong, R., \& Thongboonkerd, V. (2018). Differential proteomics of lesional vs. non-lesional biopsies revealed non-immune mechanisms of alopecia areata. Scientific Reports. 10.1038/s41598-017-18282-1

Tsai, T. F., Choi, G. S., Kim, B. J., Kim, M. B., Ng, C. F., Kochhar, P., Jasper, S., Brotherton, B., Orban, B., \& Lulic, Z. (2018). Prospective randomized study of sexual function in men taking dutasteride for the treatment of androgenetic alopecia. The Journal of Dermatology. 10.1111/1346-8138.14329.

Wang, X., Xiong, C., Zhang, L., Yang, B., Wei, R., Cui, L., \& Xing, X. (2018). Psychological assessment in 355 Chinese college students with androgenetic alopecia. Medicine. 10.1097/MD.0000000000011315

Zhang, Y., Xu, J., Jing, J., Wu, X., \& Lv, Z. (2018). Serum Levels of Androgen-Associated Hormones Are Correlated with Curative Effect in Androgenic Alopecia in Young Men. Medical Science Monitor. 10.12659/MSM.913116 